\section{Pathological findings}

The left superior parathyroid gland measured $33 \mathrm{~mm}$ in maximum dimension and weighed $4173 \mathrm{mg}$. The cut surface was nodular and brown in colour. The left inferior parathyroid measured $5 \mathrm{~mm}$ in maximum dimension and weighed $120 \mathrm{mg}$. Microscopy showed nodular hyperplasia of chief cells and oxyphil cells in both glands. Areas of haemorrhage and cystic degeneration were present in the larger left superior gland. Hobnail cells with vesicular nuclei and prominent nucleoli lined one of these cysts. The cyst lumen contained a few malignant glands. A diagnosis was made of parathyroid hyperplasia of both glands with metastatic adenocarcinoma within the left superior parathyroid. The right submandibular lymph node measured $12 \mathrm{~mm}$ in diameter and was totally replaced by metastatic poorly differentiated carcinoma with a stroma rich in osteoclastic giant cells. The tumour cells had vesicular nuclei and prominent nucleoli similar to that of the metastatic carcinoma in the left parathyroid gland but neither glandular nor squamous differentiation was evident. Immunohistochemical staining showed the tumour cells staining positively with CK7, PE10 and TTF-1, within both the lymph node and the left superior parathyroid gland. There was no reactivity to WT-1, CA-125, CK20, and HMB-45. The immunoprofile suggested a diagnosis of primary bronchogenic carcinoma with metastasis to the right submandibular lymph node and the left superior parathyroid gland (fig 1).

\section{Discussion}

We have reported a patient with primary hyperparathyroidism due to parathyroid hyperplasia and coincidental metastatic adenocarcinoma involving one of the enlarged parathyroid glands. While there are independent reports of metastatic tumour involving the parathyroids and primary hyperparathyroidism associated with disseminated malignancy, a literature search revealed no reported case similar to ours wherein the metastatic adenocarcinoma was identified as a result of parathyroid gland excision for hyperparathyroidism.

Metastasis of tumour to the parathyroid glands has been described previously in autopsy studies of known cancer patients with widespread tumour. ${ }^{12}$ The commonest sites of primary tumour in these circumstances were breast, lung, and soft tissue, also leukaemia and cutaneous melanoma. ${ }^{3}$ Secondary involvement of the parathyroid gland by local invasion of thyroid and laryngeal carcinomas is also reportedly infrequent. ${ }^{5}$ Tang et al have described involvement of the parathyroid gland by papillary thyroid carcinoma in 20 of 911 cases; $2 \%$ of these had metastases as opposed to direct invasion. ${ }^{6}$ Our case therefore appears to be unique in that the patient presented with symptoms related to hypercalcaemia and the primary lung tumour was undetected prior to removal of the parathyroid glands.

The association of hypercalcaemia with nonparathyroid cancer is well recognised. The mechanisms of the hypercalcaemia include osteolytic metastasis and hypercalcaemia developing as a non-metastatic phenomenon consequent to production of a parathyroid hormone-like humoral agent by the tumour. ${ }^{58}$ There are several case reports of hypercalcaemia due to primary hyperparathyroidism associated with cancer of the lung, and non-medullary carcinoma of the thyroid, breast and larynx, but the frequency of this association is not precisely known. Many of these cancer patients had hypercalcaemia in the absence of metastatic disease; the presence of raised parathyroid hormone levels led to evaluation of the parathyroid glands. ${ }^{9-13}$ Godsall et al noted primary hyperparathyroidism with concomitant non-parathyroid cancer in 8 of 133 patients with disseminated cancer. The types of cancer included squamous lesions of the head and neck, lung, and colon, breast adenocarcinoma and myeloma. ${ }^{14}$ Honda et al reported primary hyperparathyroidism occurring in association with aldosterone-producing adrenocortical adenoma and breast cancer, and suggested relation to MENl gene mutations. ${ }^{15}$ However, there are others who suggest that the occurrence of the two diseases is coincidental rather than due to a shared aetiology. ${ }^{13}$ Primary hyperparathyroidism due to parathyroid adenoma has also been reported with a slightly increased frequency in non-aggressive breast cancer patients. ${ }^{9}{ }^{10}$ In our patient, despite the gross impression of a parathyroid adenoma, microscopy of the left superior and inferior parathyroid glands showed hyperplasia with asymmetric enlargement of the left superior parathyroid gland. The serum calcium reverted to normal after parathyroid exploration. It seems probable that removal of the larger parathyroid gland was responsible for normalisation of the serum calcium and that the microscopic hyperplasia of the second parathyroid gland was functionally insignificant.

In conclusion, this report describes the rare incidental discovery of metastatic adenocarcinoma within a hyperplastic parathyroid gland resected for surgical management of primary hyperparathyroidism. It is important for clinicians and pathologists to be aware of this possibility in patients evaluated for osteoporosis and hypercalcaemia.

\section{Acknowledgements}

We thank Dr Seamus Napier and $\mathrm{Mr}$ Craig McLaughlin for assistance with taking the photographs.

L Venkatraman Department of Histopathology, Royal Victoria Hospital
Belfast, Northern Ireland

A Kalangutkar, C F Russell

Department of Endocrine Surgery, Royal Victoria Hospital, Belfast, Northern Ireland

Correspondence to: $\operatorname{Dr} L$ Venkatraman, Department of Histopathology, Royal Group of Hospitals Trust, Grosvenor Road, Belfast BT12 6BL, Northern Ireland

doi: 10.1136/jcp.2005.035352

Accepted 30 January 2006

Competing interests: None.

\section{References}

1 Gattuso P, Khan NA, Jablokow VR, et al. Neoplasms metastatic to parathyroid glands. South Med J 1988:81:1467.

2 Horwitz CA, Myers WP, Foote FW Jr. Secondary malignant tumors of the parathyroid gland. Report of 2 cases with associated hypoparathyroidism. Am J Med 1972;52:797-808.

3 De la Monte SM, Hutchins GM, Moore GW Endocrine organ metastases from breast carcinoma. Am J Pathol 1984;114:131-6.

4 Sidhu S, Neill AK, Russell CFJ. Long-term outcome of unilateral parathyroid exploration for primary hyperparathyroidism due to presumed solitary adenoma. World J Surg 2003;27:339-42.

5 DeLellis RA. Miscellaneous lesions. In: DeLellis RA eds. Atlas of tumor pathology. Tumors of the parathyroid gland. Washington, DC: Armed Forces Institute of Pathology, 1993:93-4.

6 Tang W, Kakudo K, Nakamura Y, et al. Parathyroid gland involvement by papillary carcinoma of the thyroid gland. Arch Pathol Lab Med 2002;26:1511-14

7 Stewart AF. Clinical practice. Hypercalcemia associated with cancer. N Engl J Med 2005;352:373-9.

8 Solimando DA. Overview of hypercalcemia of malignancy. Am J Health Syst Pharm 2001;58(Suppl 3):4-7.

9 Matsumoto J, Kojima T, Shimizu T, et al. A case of lung cancer with hypercalcemia which was incidentally complicated with primary hyperparathyroidism due to parathyroid adenoma. Ann Thorac Cardiovasc Surg 2002;3:151-3.

10 Attie JN, Vardhan R. Association of hyperparathyroidism with nonmedullary thyroid carcinoma: review of 31 cases. Head Neck 1993;1:20-3.

11 Kara IO, Sahin B, Yapar Z. Breast cancer and concomitant primary hyperparathyroidism: description of two patients. Acta Med Austriaca 2004;3:81-4.

12 Axelrod DM, Bockman RS, Wong GY, et al. Distinguishing features of primary hyperparathyroidism in patients with breast cancer Cancer 1987;60:1620-4.

13 Haar JG, Boulos EJ. Primary hyperparathyroidism and laryngeal carcinoma: a cause of associated hypercalcemia. Laryngoscope 1981;11:1937-40.

14 Godsall JW, Burtis WJ, Insogna KL, et al. Nephrogenous cyclic AMP, adenylate cyclase stimulating activity and the humoral hypercalcemia of malignancy. Recent Prog Horm Res 1986:42:705-50

15 Honda M, Tsukada T, Horiuchi T, et al. Primary hyperparathyroidism associated with aldosteroneproducing adrenocortical adenoma and breast cancer: relation to MEN1 gene. Intern Med 2004;43:310-14.

\section{Granulomatous reaction to injectable hyaluronic acid (Restylane) diagnosed by fine needle biopsy}

The hyaluronic acid (HA) derivative Restylane is now the most common injectable soft tissue filler used for facial wrinkle augmentation. Although it is generally well tolerated and absorbed within months, nodule formation at the injection site has been documented.

A 62-year-old woman with a history of carcinoma of the breast six years previously was referred to a fine needle aspiration (FNA) clinic for biopsy of a subcutaneous facial nodule. On palpating the nodule six weeks prior to presentation she was referred for ultrasound examination; findings were reported as being consistent with a lymph node, raising concern of possible metastatic carcinoma of the breast. Serendipitously the same ultrasound examination led to discovery of a thyroid nodule that was aspirated and found to be papillary carcinoma, broadening the differential diagnosis of the facial nodule to metastatic thyroid carcinoma.

On physical examination the lesion was a $1 \mathrm{~cm}$ subcutaneous nodule overlying the lower third of the mandible, a location felt to be consistent with a slightly "high" submental node. However, the nodule had irregular contours on palpation, and although it could be moved over the underlying bone, seemed to be fixed to the overlying skin. No cellular material was obtained by initial sampling with 
a $27 \mathrm{G}$ needle. After instillation of local anaesthetic, two samples were taken with a $23 \mathrm{G}$ needle using Zajdela's technique. ${ }^{1}$ Direct smears were made for Diff-Quik (Harleco, New Jersey, USA) staining; the needle was rinsed in CytoLyt (CYTYC Corp, Massachusetts, USA) for preparation of a Papanicolaou stained Thin-Prep (CYTYC Corp, Massachusetts, USA) slide. Two cytospin slides were kept for potential immunoperoxidase staining.

Microscopic examination of the Diff-Quik stained sample showed a highly cellular specimen composed of two elements: histiocytes which were mainly aggregated to form foreign body type giant cells, and amorphous foreign material which stained a deep magenta colour on Diff-Quik (fig 1). Staining of the foreign material, which was present in rounded masses measuring up to $300 \mu \mathrm{m}$ in diameter, was homogeneous in some fragments, but granular material suggestive of degeneration or possibly calcium deposition was present in others. Some foreign material was seen lying free in the background of the smear, but most of it was seen in juxtaposition to giant cells, which appeared to be engulfing it (fig 2). Some giant cells showed "tears" in their cytoplasm which may have been caused during smear preparation; phagocytosed foreign material lagged behind as the surrounding cell was smeared flat. There was no evidence of malignancy, or of a chronic lymphocytic or suppurative inflammatory response.

Although paucicellular, the Papanicolaou stained Thin-Prep slide recapitulated the findings of giant cells and foreign material seen on the air-dried Diff-Quik stained material. In the Papanicolaou stain the foreign material appeared light grey in colour, finely granular in consistency, and showed a slightly "frayed" edge. Occasional histiocytes were embedded in this material.

Further questioning of the patient revealed injection of Restylane at the site of the nodule ten months before. Because of the referring surgeon's concern that the observed acellular material might be thyroglobulin eliciting a giant cell response, the reserved cytospin slides were used for immunohistochemical staining for TTF1 and thyroglobulin (Ventana: TTF-1 clone 8G7G3/1, thyroglobulin clone $2 \mathrm{H} 1 \mathrm{l} / 6 \mathrm{El}$ ). These stains were completely negative. In view of the bland typical histiocytic morphology, it was felt unnecessary to restain these slides for CD68 and pan-cytokeratin. Staging investigations undertaken before the patient's thyroid surgery did not

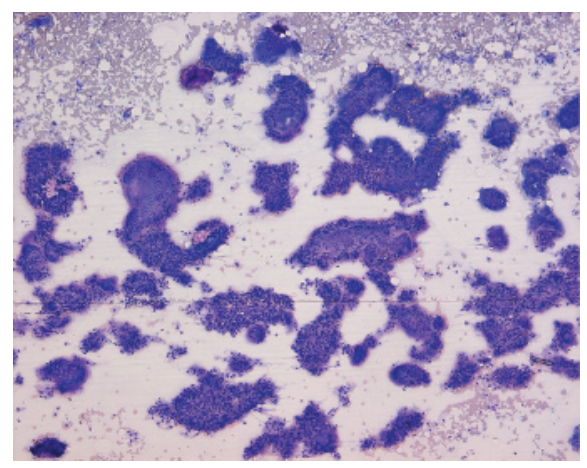

Figure 1 Diff-Quik stained smear showing foreign body giant cells, foreign material and dispersed histiocytes (original magnification, $\times 100)$.

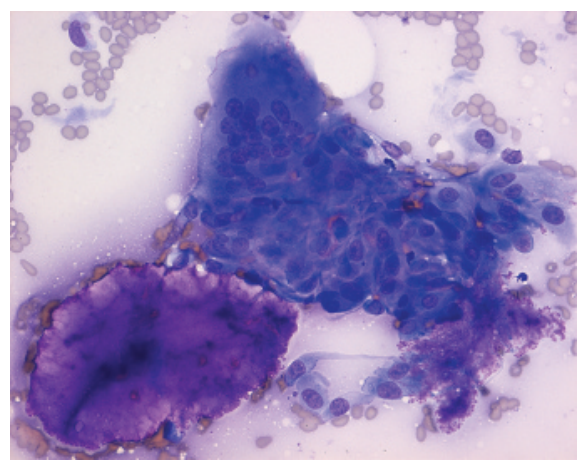

Figure 2 Diff-Quik stained smear contains rounded mass of Restylane with adjacent giant cell (original magnification, $\times 400$ ).

reveal any evidence of recurrent breast carcinoma. Thyroidectomy confirmed a $1.6 \mathrm{~cm}$ papillary carcinoma limited to the thyroid. Six months after the needle biopsy the patient reports that the facial nodule has completely resolved.

\section{Discussion}

HA derivatives such as Restylane are among the most utilised soft tissue filler substances, principally being used as a non-surgical cosmetic option for skin augmentation to correct age related skin facial wrinkling, or for treatment of post-surgical and post-traumatic skin defects.

Restylane is manufactured by bacterial fermentation techniques, and its biological characteristics generally allow high tolerance However, it is not totally non-immunogenic and is said to be contraindicated in patients with autoimmune diseases, or patients taking immunosuppressant therapy. ${ }^{3}$ The short-term adverse effects of injection may include a transient acute inflammatory response in about $4-5 \%$ of patients, manifested clinically as redness, bruising, pain and swelling localised to the site of the injection. ${ }^{4}{ }^{8} \mathrm{~A}$ few reports describe a delayed inflammatory response consisting of a granulomatous foreign body reaction occurring at the site of the injection. ${ }^{245}$ The aetiology of this granulomatous reaction has been ascribed to protein impurities resulting from the bacterial fermentation. ${ }^{6}$ To the best of our knowledge this is the first report of diagnosis of this reaction by fine needle aspiration biopsy. In addition to illustrating the feasibility of FNA diagnosis of Restylane granuloma, this case highlights the value of early cytological evaluation of a palpable lesion, especially with immediate assessment of a smear while the patient is still available for questioning. The information obtained is frequently sufficient to correct erroneous conclusions arising from non-specific imaging findings, rule out other clinically suspected lesions and point to the correct line of inquiry required for diagnosis.

The strong magenta coloration of the Restylane we observed on Diff-Quik staining could conceivably be mistaken for rounded bodies of basement membrane material, colloid, or mucin, prompting differential diagnoses of various salivary gland, thyroid, or breast neoplasms, but those possibilities are easily ruled out by the absence of any observed epithelial component, or, if necessary, by staining for cytokeratin. The finding of a foreign body giant cell reaction in FNA material from a facial lesion should prompt further questions about use of injectable filler substances in the area. As this case illustrates, the granulomatous reaction that occasionally occurs with these materials may only be recognised by the patient some months after their injection, and history of their use may not be offered initially.

Cytopathologists or pathologists seeing similar lesions might be questioned about appropriate treatment. Surgical removal is curative but may be of cosmetic concern to the patient. Local injection of steroids has been used with some success. ${ }^{7}$ Our patient was managed conservatively without the use of steroids, and the nodule has disappeared. It is possible that the partial removal of the foreign material by the needle biopsy may have accelerated the resolution of the lesion.

Mubarak Al-Shraim, Mohammad Jaragh, William Geddie

Department of Laboratory Medicine and Pathobiology, University of Toronto, Toronto, Ontario, Canada

Correspondence to: Dr William Geddie, Department of Laboratory Medicine and Pathobiology, University Health Network, 200 Elizabeth street, 11 E219, Toronto, Ontario M5G 2C4, Canada; william.geddie@úhn.on.ca

doi: 10.1136/jсp.2007.048330

Accepted 5 April 2007

Competing interests: None declared

\section{References}

1 Zadela A, deMaüblanc MT, Schlienger $P$, et al. Cytologic diagnosis of periorbital palpable tumours using fine-needle sampling without aspiration. Diagn Cytopathol 1986;2:17-20.

2 Jordan DR. Delayed inflammatory reaction to hyaluronic acid (Restylane) Ophthal Plast Reconstr Surg 2005;21:401-2.

3 Ghislanzoni M, Bianchi F, Barbareschi $M$, et al. Cutaneous granulomatous reaction to injectable hyaluronic acid gel. Br J Dermatol 2006;154:755-8.

4 Duranti F, Salti G, Bovani B, et al. Injectable hyaluronic acid gel for soft tissue augmentation. A clinical and histological study. Dermatol Surg 1998;24:1317-25

5 Rongioletti F, Cattarini G, Sottofattori E, et al. Granulomatous reaction after intradermal injections of hyaluronic acid gel. Arch Dermatol 2003;139:815-16.

6 Friedman PM, Mafong EA, Kauvar AN, et al. Safety data of injectable nonanimal stabilized hyaluronic acid gel for soft tissue augmentation. Dermatol Surg 2002;28:491-4.

7 Shafir R, Amir A, Gur E. Long-term complications of facial injections with Restylane injectable hyaluronic acid. Plast Reconstr Surg 2000;106:1215-16.

\section{Follicular lymphoma with trisomy 18 exhibiting loss of BCL-2 expression on transformation to a large cell lymphoma}

Over-expression of BCL-2 in follicular lymphoma is commonly caused by a $\mathrm{t}(14 ; 18)$ chromosomal translocation. Follicular lymphomas that do not carry the $\mathrm{t}(14 ; 18)$ translocation but are positive for BCL-2 protein may have other cytogenetic abnormalities, including BCL-2 amplification, reciprocal translocations involving the $I G K / I G L \operatorname{loci}^{1}$ and trisomy $18 .^{23}$

Transformation of follicular lymphoma to diffuse large B cell lymphoma is generally associated with a poorer prognosis. ${ }^{45}$ However, 